\title{
ÉTUDE DE LA SORPTION D'HYDROCARBURES AROMATIQUES POLYCYCLIQUES EN MILIEUX POREUX NATURELS SATURÉS PAR CHROMATOGRAPHIE ELUTO-FRONTALE
}

\author{
Jean-Christophe Appert-Collin*, Marie-Odile Simonnot** et Michel Sardin*** \\ Laboratoire des sciences du génie chimique, CNRS, Ensic INPL
}

La rétention monoconstituant de trois HAP (naphtalène, fluorène, phénanthrène) par deux sables, l'un contenant $2 \%$ de carbone organique, l'autre imprégné d'un hydrocarbure saturé, est étudiée par des expériences en colonnes saturées, selon l'approche de la chromatographie éluto-frontale. Les expériences en colonne mettent bien en évidence la non-linéarité du processus de partage entre la matière organique du sable et l'eau. La rétention par le sable organique est décrite par une isotherme de Langmuir, dont la pente à l'origine peut être prédite par la loi de Karickhoff. L'isotherme de rétention par le sable imprégné présente une courbure convexe, ce qui indique que la rétention est renforcée en présence d'un hydrocarbure non polaire. Ainsi, les HAP ont tendance à être peu mobiles en présence d'autres polluants peu polaires. La détermination quantitative des paramètres d'équilibre permet de prévoir le transport de ces HAP en milieux poreux notamment lors d'expériences de lixiviation en colonnes pilotes.

The individual retention of three PAH (naphtalene, fluorene and phenanthrene) by two sands was studied by mean of column experiments, following the methods of frontal chromatography. The first sand naturally contained $2 \%$ organic carbon, as the second one was coated with a heavy $\mathrm{Cl} 6$ apolar hydrocarbon. Column experiments show that retention is non linear, since the breakthrough curve location depends on the PAH feed concentration, and sorption and regeneration fronts are more or less sharpening or broadening, following the retention isotherm convexity. Retention by the organic sand follows a Langmuir isotherm and the initial slope is in good agreement with the prediction of the Karickhoff law. Retention on the coated sand can be described by a Freundlich isotherm with an exponent slightly greater than unity. Thus, retention is enhanced if a nonpolar hydrocarbon is present, that means that HAP propagation is lowered. The quantitative determination of equilibrium interaction parameters enables us to predict HAP transport through porous media, e.g. the results of lixiviation experiments at the pilot scale.

\section{INTRODUCTION}

Le recensement des sites pollués effectué par l'Ademe ${ }^{[l]}$ montre que les sols des anciennes usines à gaz et cokeries sont contaminés par des hydrocarbures aromatiques polycycliques (HAP) souvent associés à d'autres polluants organiques et à des métaux. Potentiellement dangereux, car pour certains mutagènes et cancérigènes, les HAP ne constituent un risque que dans la mesure où ils sont susceptibles d'atteindre une cible sensible : écosystème fragile, ressource en eau, alimentation animale ou humaine. Les vecteurs du transport sont multiples : contact ou ingestion directs, transport aérien, transport par l'eau d'infiltration. Dans ce dernier cas, la quantité de HAP mobilisée lors de la lixiviation d'un site pollué dépend à la fois de la capacité du sol contaminé à libérer les HAP et des propriétés de rétention des zones non contaminées que l'eau chargée en HAP va rencontrer au cours de son transit. Le transport d'un polluant retenu par un milieu poreux naturel est alors régi par le couplage entre :

- l'équilibre thermodynamique, par exemple le partage d'un constituant entre la phase aqueuse et les phases solides, constituées de fractions minérales et de fractions organiques comportant des matières naturelles et/ou exogènes,

- la cinétique des réactions chimiques et biochimiques de dégradation du polluant,

- les cinétiques de transfert entre phases liées aux processus diffusionnels,

- l'hydrodynamique (transport advectif et dispersif) qui règlent le transport à longue distance.

Les principales difficultés rencontrées pour prédire le transport de HAP en milieu poreux naturel résident dans (i) la détermination précise des lois de partage en tenant compte de la complexité du milieu poreux, (ii) l'établissement des liens entre structure géométrique du milieu et propriétés des coefficients de transport et (iii) la détermination des lois macroscopiques qui gouvernent le mouvement des polluants à l'échelle du terrain. Aussi la prévision des conséquences de la pollution localisée d'un sol par des HAP restet-elle une question ouverte.

Les méthodes de la chromatographie ${ }^{[2]}$ sont bien adaptées 
pour aborder ce type de problème, aussi bien pour l'étude des interactions polluant-terre que pour la description et la prédiction des résultats de lixiviation voire de lessivage. Le couplage entre les expériences en colonne de laboratoire et la modélisation du transport constitue un moyen d'investigation qui conduit à des informations d'une grande sûreté. La méthodologie d'étude consiste à la mise en place du milieu poreux dans une colonne de laboratoire aux dimensions adaptées au problème posé. On sature la colonne en eau et on assure un régime d'écoulement contrôlé. La colonne est alors soumise en entrée à des signaux de concentration dont la déformation est analysée en sortie grâce à des techniques de mesure adaptées, si possible en ligne, sinon après collecte de fractions. On obtient ainsi des courbes de percée dont la position moyenne et la forme fournissent des informations sur les interactions subies par le soluté au sein du milieu. Éventuellement après la percée, l'analyse des profils de concentrations à l'intérieur de la colonne après découpage de la colonne permet d'accéder à des informations complémentaires sur les solutés les plus fortement retenus. La comparaison des courbes de percée avec les simulations issues d'un modèle phénoménologique décrivant macroscopiquement les interactions permet de quantifier et de valider les modèles proposés.

Cette approche est illustrée ici par la présentation d'une partie des travaux menés en collaboration avec l'Ademe et I'IRH Environnement dont l'objectif était d'évaluer les risques de propagation d'une contamination par les HAP en étudiant le transport par l'eau et la sorption sur des échantillons de sols de ces polluants ${ }^{[3]}$. La démarche globale a consisté à partir de l'étude de la sorption monoconstituant de HAP sur des milieux poreux modèles ${ }^{[4]}$ pour aller jusqu'à la lixiviation d'échantillons de sols de cokerie fortement pollués à l'échelle pilote. La première étape décrite dans cet article est la quantification des propriétés de transport monoconstituant de trois HAP « légers » (naphtalène, fluorène et phénanthrène) dans des milieux poreux naturels modèles à teneur en matière organique connue.

\section{MATÉRIELS ET MÉTHODES}

\section{Les milieux poreux}

Deux sables ont été utilisés : un sable organique $\left(84 \% \mathrm{SiO}_{2}\right.$, $4 \%$ argiles, $2 \%$ calcite, $2 \%$ carbone organique) et un sable minéral ( $95 \% \mathrm{SiO}_{2}, 3 \%$ argiles, I \% calcite) imprégné d'un hydrocarbure saturé en $\mathrm{Cl} 6$, le 2,2,4,4,6,8,8Heptaméthylnonane (HMN), à 0,5 ou $0,33 \%$ en poids, soit respectivement 0,42 et $0,28 \%$ de carbone organique. Les sables sont initialement secs, tamisés à $250 \mu \mathrm{m}$ et le diamètre moyen des grains est de $170 \mu \mathrm{m}$.

\section{Les solutions d'alimentation}

Les HAP (tableau I) sont dissous individuellement dans une solution de fond qui est une solution aqueuse de $\mathrm{CaCl}_{2} 10^{-3} \mathrm{~mol}$. $\mathrm{L}^{-1}$, pour éviter de modifier les caractéris-
Tableau I : Caractéristiques physico-chimiques des HAP utilisés

\begin{tabular}{lcccc} 
Formule & $\begin{array}{c}\text { Masse } \\
\text { molaire } \\
\left(\mathbf{g} \cdot \mathbf{m o l}^{-1}\right)\end{array}$ & $\begin{array}{c}\text { Coefficient de } \\
\text { partage octanol/ } \\
\text { eau (Log Kow) }\end{array}$ & $\begin{array}{c}\text { Solubilité } \\
\text { dans l'eau à } \\
\mathbf{2 0} 0^{\circ} \mathbf{C}\left(\mathbf{m g} \cdot \mathbf{L}^{-1}\right)\end{array}$ \\
\hline Naphtalène & $\mathrm{C}_{10} \mathrm{H}_{8}$ & 128,2 & 3,37 & 31 \\
Fluorène & $\mathrm{C}_{13} \mathrm{H}_{10}$ & 166,2 & 4,18 & $\mathrm{I}, 9$ \\
\hline Phénanthrène $\mathrm{C}_{14} \mathrm{H}_{10}$ & 178,2 & 4,57 & $\mathrm{I}, \mathrm{I}$
\end{tabular}

tiques du milieu par échange cationique et de déstabiliser les argiles.

La très faible solubilité des HAP dans l'eau nécessite un cosolvant (méthanol) pour la préparation des solutions-mères dont la présence ( $<2 \%$ vol.) ne modifie pas les propriétés de sorption des HAP sur les matériaux étudiés ${ }^{[5]}$.

\section{Montage expérimental}

C'est un montage de chromatographie HPLC comprenant une pompe à pistons, une colonne en inox $(\mathrm{L}=10 \mathrm{~cm}$, $\varnothing=0,8 \mathrm{~cm}$, volume : $5 \mathrm{~mL}$, masse de sable : $8 \mathrm{~g}$ ) alimentée de bas en haut et une détection fluorimétrique en ligne en sortie de colonne avec acquisition de données. L'ensemble des connexions est en inox pour éviter la sorption des HAP sur des polymères comme le Téflon ${ }^{[6]}$.

\section{Protocole expérimental}

En premier lieu, un traçage du milieu poreux est effectué à l'aide de chlorure de calcium, qui est considéré comme un bon traceur de l'eau dans les milieux poreux dont la composition ionique de surface et le $\mathrm{pH}$ sont contrôlés par les équilibres calcocarboniques. Le traçage consiste à mesurer en ligne (par conductimétrie) la réponse de la colonne alimentée par un échelon de $\mathrm{CaCl}_{2}$ de $10^{-3}$ à $10^{-2} \mathrm{~mol}$. $\mathrm{L}^{-1}$. Le débit est de $1 \mathrm{~mL} . \mathrm{mn}^{-1}$, la vitesse linéaire u de $3,3210^{-4} \mathrm{~m} \cdot \mathrm{s}^{-1}$.

Ensuite, les expériences monoconstituants consistent à injecter dans la colonne jusqu'à saturation une solution de concentration $\mathrm{C}_{0}$ selon un signal échelon, puis à effectuer la désorption selon une purge-échelon, en injectant la solution de fond. La concentration en HAP dans l'effluent est suivie par fluorimétrie en continu. Cette expérience est effectuée à différentes concentrations pour chaque HAP et pour chaque sable.

\section{Analyse des courbes de percée}

La courbe de percée du traceur donne le volume poreux accessible au fluide ainsi que la dispersion hydrodynamique $D_{H}$ ce qui permet de modéliser l'écoulement de l'eau. Pour toutes les expériences, le volume poreux est de $2,7 \mathrm{~mL}$ et le nombre de Péclet $\left(=\mathrm{uL} / \mathrm{D}_{\mathrm{H}}\right)$ est de l'ordre de I00. L'analyse de la famille des courbes de percée d'un constituant donnant $\mathrm{C} / \mathrm{C}_{0}$ en fonction du volume réduit de solution passé à travers la colonne $\mathrm{V} / \mathrm{VP}$ et obtenues à plusieurs concentrations d'alimentation permet de remonter à l'isotherme d'équilibre liquide-solide. En supposant que la saturation de la colonne correspond à l'équilibre, le calcul de la surface comprise entre la courbe de saturation et la droite $\mathrm{C} / \mathrm{C}_{0}=1$ 
donne la quantité fixée à l'équilibre pour la concentration $\mathrm{C}_{0}$, soit un point de l'isotherme de sorption. L'isotherme est alors construite point par point. Au préalable, la comparaison entre les masses de HAP fixée et libérée lors de la purge-échelon aura montré la réversibilité du processus de sorption.

L'analyse se poursuit en étudiant la forme des courbes de percée, information plus délicate à exploiter car la forme des courbes dépend de plusieurs processus qu'il n'est pas toujours aisé de dissocier sans l'aide d'un modèle numérique : équilibre, cinétiques et advection-dispersion. Toutefois, la linéarité de l'interaction peut être immédiatement évaluée en comparant le front de saturation et la courbe obtenue en transformant la réponse à la purge-échelon en $\mathrm{I}-\mathrm{C} / \mathrm{C}_{0}$ (figures Ib et $3 b$ ). La superposition des courbes traduit un processus linéaire, tandis que leur non-superposition indique un processus non linéaire. Cette observation est d'autant plus aisée que la colonne est peu dispersive et que l'équilibre local est atteint. Dans ce cas, on peut extraire de la courbe réponse à la purge-échelon (isotherme concave) ou à l'échelon (isotherme convexe) la branche d'isotherme comprise entre la concentration initiale d'injection et la concentration finale $e^{[2]}$

\section{RÉSULTATS ET DISCUSSION}

Des études préliminaires ${ }^{[[]}$ont montré que l'adsorption des HAP sur les surfaces minérales en milieu aqueux saturé était négligeable par rapport à la rétention par la matière organique.

\section{Sable organique des Pays-Bas}

La figure I montre le réseau de courbes de percée obtenues avec le phénanthrène ainsi que la comparaison entre une courbe de saturation et la transformée de la courbe de régénération. La non linéarité de la sorption apparaît clairement. On en déduit l'isotherme de sorption comme décrit précédemment. Des résultats analogues ont été obtenus pour le naphtalène et le fluorène $\mathrm{e}^{[3]}$ (figure 2). Ces isothermes obéissent au formalisme de l'isotherme de Langmuir (tableau 2) :

$q=\frac{K_{L} S_{\circ} C}{I+K_{L} C}$

$C$ et $q$ sont respectivement la concentration à l'équilibre en solution du soluté étudié en $\mathrm{Mol} / \mathrm{L}^{-1}$ et la quantité fixée (ou sorbée) sur le milieu en $\mathrm{Mol} / \mathrm{kg}^{-1}$ de sol ou de matière organique. Les paramètres de l'isotherme sont la constante de l'équilibre de sorption, $K_{L}$ en $L / \mathrm{Mol}^{-1}$, et $S_{0}$, la quantité maximale sorbable en $\mathrm{Mol} / \mathrm{kg}^{-1}$. On privilégie cette représentation par rapport au modèle de Freundlich parce qu'elle restitue mieux la linéarité observée aux faibles concentrations (<
Tableau 2 : Coefficients des paramètres de l'équation de Langmuir et comparaison de la pente à l'origine au coefficient de distribution prédit par la loi de Karickhoff

\begin{tabular}{|c|c|c|c|c|}
\hline & $\begin{array}{c}\mathrm{K}_{\mathrm{L}} \\
\text { (L. } \mathrm{mol}^{-1} \text { ) }\end{array}$ & $\begin{array}{c}S_{0} \\
\left(\mathrm{~mol}^{\prime} \mathrm{kg}^{-1}\right)\end{array}$ & $\begin{array}{c}\mathrm{K}_{\mathbf{L}} \mathrm{S}_{0} \\
\left(\mathrm{~L} . \mathrm{kg}^{-1}\right)\end{array}$ & $\begin{array}{c}K_{d}=f_{o c} K_{o c} \\
\left(L . k^{-1}\right)^{*}\end{array}$ \\
\hline Naphtalène & 5095 & $2,910^{-3}$ & 15 & 19 \\
\hline Fluorène & 108000 & $1,110^{-3}$ & 119 & 123 \\
\hline Phénanthrène & 90700 & $3,210^{-3}$ & 290 & 298 \\
\hline
\end{tabular}

* Calculé par : $\log \mathrm{K}_{\mathrm{oc}}=0.989 \log \mathrm{K}_{\mathrm{ow}}-0.346^{[7]}$

$20 \%$ de la solubilité). Dans ce domaine, l'isotherme devient $q=K_{L} S_{0} C$ et le produit $K_{L} S_{0}$ peut être comparé au coefficient de distribution couramment employé dans la littérature ${ }^{[7,8]} K_{d}=f_{o c} K_{o c}$ (loi de Karickhoff), où $f_{o c}$ est la fraction de carbone organique du solide. $K_{o c}$ est prédit par des corrélations du type : $\log \mathrm{K}_{o c}=\mathrm{a} \log \mathrm{K}_{\mathrm{ow}}+\mathrm{b}$ dans lesquelles $\mathrm{K}_{\text {ow }}$ est le coefficient de partage du soluté entre l'eau et l'octanol et

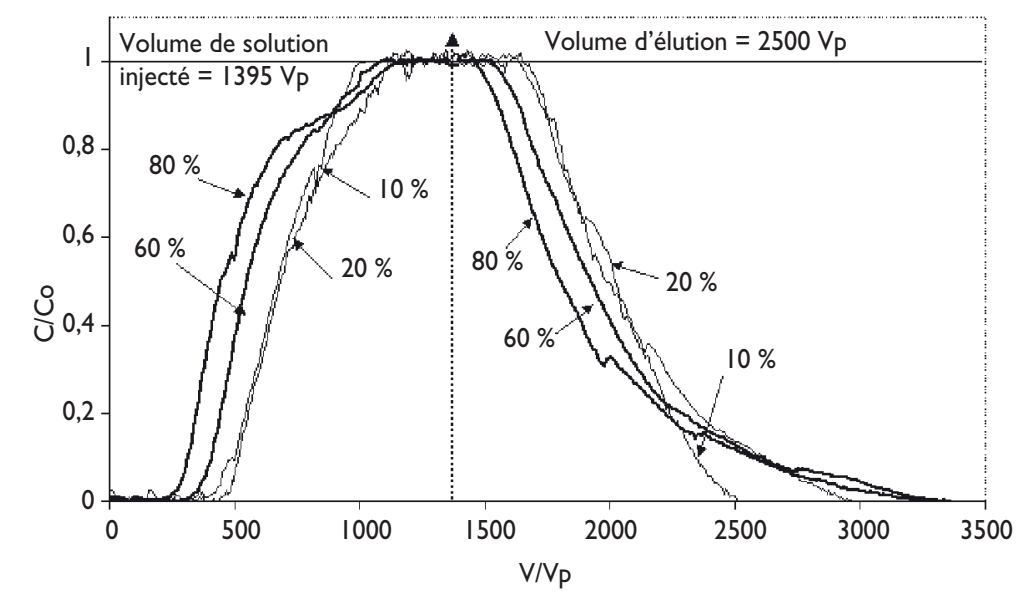

(a) Courbes de percée du phénanthrène. Les courbes à $10 \%$ et $20 \%$ sont très proches indiquant un processus linéaire dans ce domaine

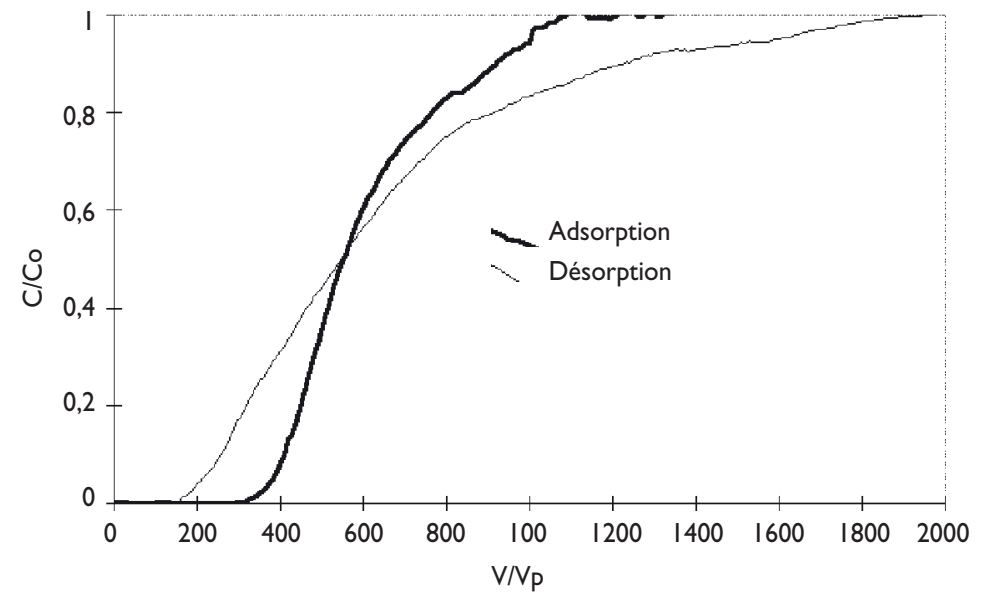

(b) Comparaison des courbes d'adsorption et de désorption modifiée $(\mathrm{I}-\mathrm{C} / \mathrm{CO})$ du phénanthrène à une concentration de $60 \%$ de sa solubilité dans l'eau mettant en évidence la non-linéarité

Figure I : Sable organique 


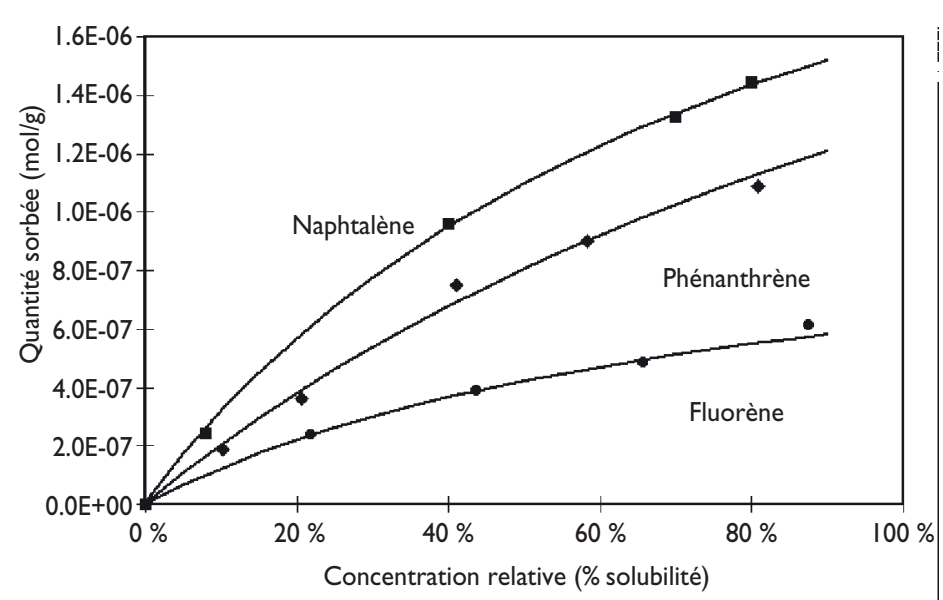

(a) sable organique

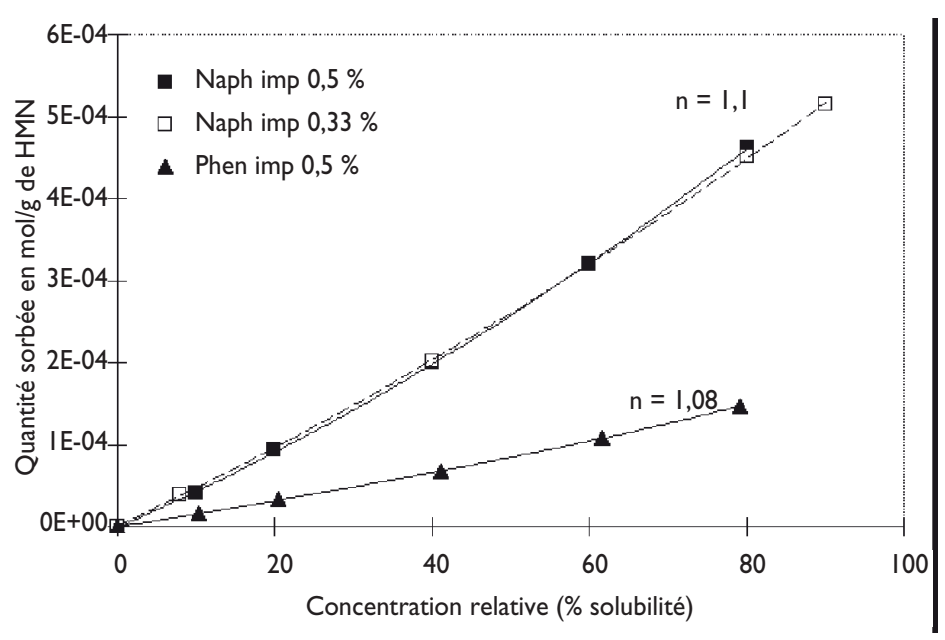

(b) sable imprégné d'HMN

Figure 2 : Exemples d'isothermes d'équilibre déduit des courbes de percée à $20^{\circ} \mathrm{C}$

$a$ et $b$ des coefficients empiriques. L'accord entre $K_{L} S_{0}$ et $K_{d}$ est très bon (tableau 2).

\section{Sable imprégné d'heptaméthylnonane}

La figure 3 représente le réseau de courbes de percée du naphtalène sur le sable imprégné d'HMN. De nouveau, le processus de sorption est réversible, la position moyenne des courbes dépend de la concentration d'alimentation et les courbes de saturation ne sont pas superposables aux courbes de désorption transformées. Mais cette fois-ci, les courbes sont d'autant plus retardées que la concentration augmen-
Tableau 3 : Paramètres de l'isotherme de Freundlich sur le sable imprégné d'HMN

$\begin{array}{lcc} & \mathbf{K}_{\mathrm{F}}(\mathbf{m o l} / \mathbf{g ~ H M N}) /(\mathbf{m o l} / \mathbf{l})^{\mathbf{n}} & \mathbf{n} \\ \text { Naphtalène } & 5,73 & \mathrm{I}, \mathrm{I} \\ \text { Phénanthrène } & 62,3 & \mathrm{I}, 08\end{array}$

te, avec un front plus raide à la désorption qu'à la saturation. L'équilibre est non linéaire, l'isotherme est convexe et peut être représentée par le modèle de Freundlich $q=K_{F} C^{n}$ avec $n>I$ (tableau 3). La comparaison avec la loi de Karickhoff conduit à des sorptions quatre fois plus fortes que prévues. Ceci est dû à la faible polarité de la molécule d'HMN comparée à celle des molécules composant la matière organique naturelle.

Les résultats obtenus avec les différents composés montrent que la non-linéarité s'atténue à mesure que la masse molaire du composé augmente. Cependant, la méthode expérimentale de la chromatographie éluto-frontale permet de mettre en évidence sans ambiguiité la non-linéarité sur la gamme de concentration étudiée.

\section{CONCLUSION}

L'analyse des courbes de percée des HAP dans des milieux poreux naturels montre l'influence de la nature et de la quantité de matière organique sur la sorption des HAP. La non-linéarité des interactions est mise en évidence ce qui rejoint les résultats d'autres travaux ${ }^{[9]}$. Négliger cette non-linéarité conduirait à surestimer la quantité de HAP fixée par les milieux, et par conséquent à sous-estimer la mobilité et les risques de propagation de la pollution. La différence de rétention vis-à-vis des HAP entre la matière organique naturelle et la phase huile synthétique est attribuée à la différence de polarité entre ces deux matériaux. La présence d'huile réduit la mobilité des HAP, ce qui signifie que les HAP sont peu mobiles lorsqu'ils se trouvent sorbés dans une source localisée contaminée par les hydrocarbures. Ce comportement est confirmé par les résultats d'expériences biconstituants : la présence d'un second HAP tend à renforcer la rétention ${ }^{[3]}$. Ceci prouve que le mécanisme d'interaction est un processus de partage et non d'adsorption compétitive. Enfin, ces résultats ont conduit à la détermination quantitative des lois d'équilibre. Ces lois peuvent alors être utilisées grâce à des codes permettant le calcul du transport de solu-

\section{DÉCHETS SCIENCES \& TECHNIQUES}

SAP - 7, chemin de Gordes - 38100 Grenoble - Tél. : 0476432864 - Fax : 0476569409 - Mél : sap@pro-environnement.com Service abonnement : Joséphine Sambito/SAP (téléphoner du lundi au vendredi de $9 \mathrm{H}$ à $12 \mathrm{H}$ et de $13 \mathrm{H}$ à $15 \mathrm{H} 30$ ).

$\mathrm{N}^{0}$ de commission paritaire : 76929 - No ISSN : 0753-3454. Dépôt légal : 663 septembre 2000 - Imprimerie Louis Jean/Gap Photocomposition SAP

Principaux associés : Reed Exhibition Companies. 


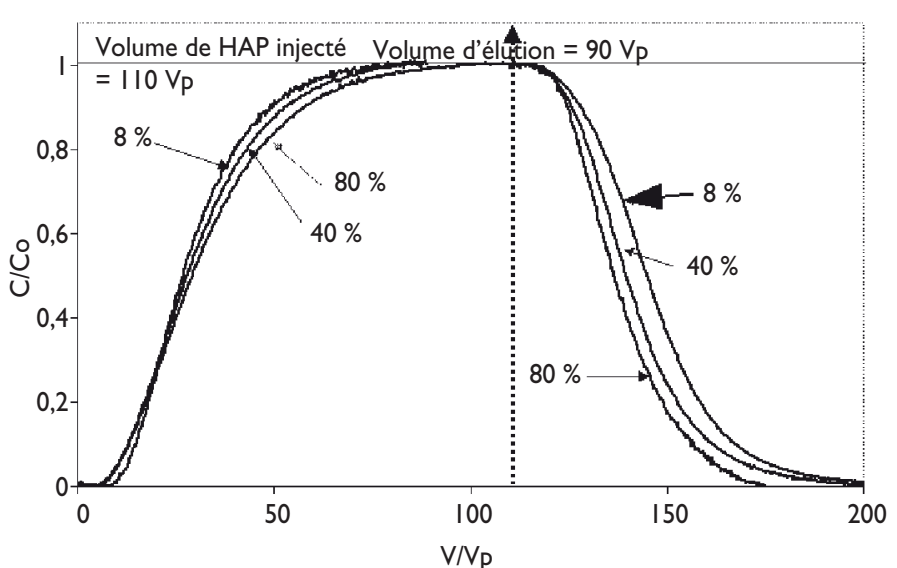

(a) Courbes de percée du naphtalène

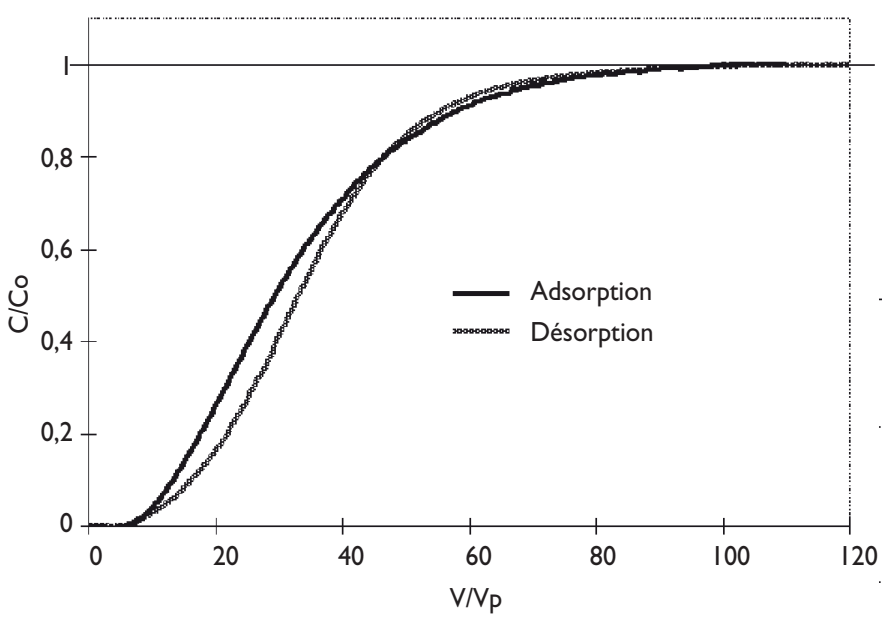

(b) Comparaison des courbes d'adsorption et de désorption modifiée $(\mathrm{I}-\mathrm{C} / \mathrm{CO})$ du naphtalène à une concentration égale à $80 \%$ de sa solubilité dans l'eau.

Figure 3 : Sable imprégné d'HMN :

tés réactifs en milieux poreux, comme le code $\operatorname{Impact}^{[10]}$. La suite de nos travaux ${ }^{[3]}$ montre que ces lois permettent de simuler presque quantitativement une expérience de lixiviation de terre polluée effectuée en colonne pilote.

\section{* Jean-Christophe Appert-Collin}

Ater à l'IUT de génie chimique de Nancy (UHP) - Laboratoire des sciences du génie chimique - I, rue Grandville - BP 45I - 5400I Nancy cedex

\section{** Marie-Odile Simonnot}

Maître de conférences à l'EEIGM (INPL) - Laboratoire des sciences du génie chimique - I, rue Grandville - BP 45I - 5400I Nancy cedex

\section{**** Michel Sardin}

Professeur à l'ENSIC (INPL) - Laboratoire des sciences du génie chimique - I, rue Grandville - BP 45I - 5400I Nancy cedex

\section{Remerciements :}

Ce travail s'inscrit dans le cadre d'une recherche financée par l'Ademe et IRH Environnement.

\section{Bibliographie}

[I] Ministère de l'Aménagement du if diagenetic processes on sorption enerterritoire et de l'Environnement getics., Environ. Sci. Technol., 29, Recensement des sites et sols pollués 92-97 (1995).

1996, (1997)

[7] Karickhoff S.W., Brown D.S.,

[2] Schweich D. and Sardin M., Scott T.A., Sorption of hydrophobic Adsorption, partition, ion exchange and pollutants on natural sediments, Wat. chemical reaction in batch reactors or Res., I 3 (3), 24I-248 (I979).

in columns - a review. Journal of Hydrology, 50, I-33 (I98I).

[8] Chiou C.T., Theoretical consideration of the partition uptake of nonio-

[3] Appert-Collin J.C., Contribution à nic organic compounds by soil organic l'analyse des risques liés au transport matter, Reactions and mouvement d'hydrocarbures aromatiques polycy- of organic chemicals in soils - SSSA, cliques dans les milieux poreux natu- special publication $n^{\circ} 22$ (1989).

rels : du système modèle à l'échantillon [9] Grathwohl P., Influence of orgade sol pollué, Thèse de Doctorat nic matter from soils and sediments INPL, Nancy (1999)

from various origins on the sorption of

[4] Appert-Collin J. C., Dridi- some chlorinated aliphatic hydrocarDhaouadi S., Simonnot M. O. and bons : implications on $K_{o c}$ correlations, Sardin M., Nonlinear sorption of naph- Environmental Science and talene and phenanthrene during satu- Technology, 24, I687-I693 (1990). rated transport in natural porous media, Phys. Chem. Earth, 24 (6), 543-549 (1999).

[10] Jauzein M., Andre C., Margrita R., Sardin M. and Schweich D., A flexible computer code

[5] Dridi-Dhaouadi S., Contribution à for modelling transport in porous l'étude du transport des hydrocarbures media : Impact, Geoderma, 44, 95polyaromatiques en milieux poreux II3 (1989).

naturels saturés, Thèse de Doctorat INPL, Nancy (I997).

[6] Young T.M., Weber W. J. Jr, A distributed reactivity model for sorption by soils and sediments. 3. Effects

Format $15^{\star} 24,104$ pages, Prix : 132 F TTC $(125,12$ F HT, TVA 5,5 \% : 6,88 F), franco de port.

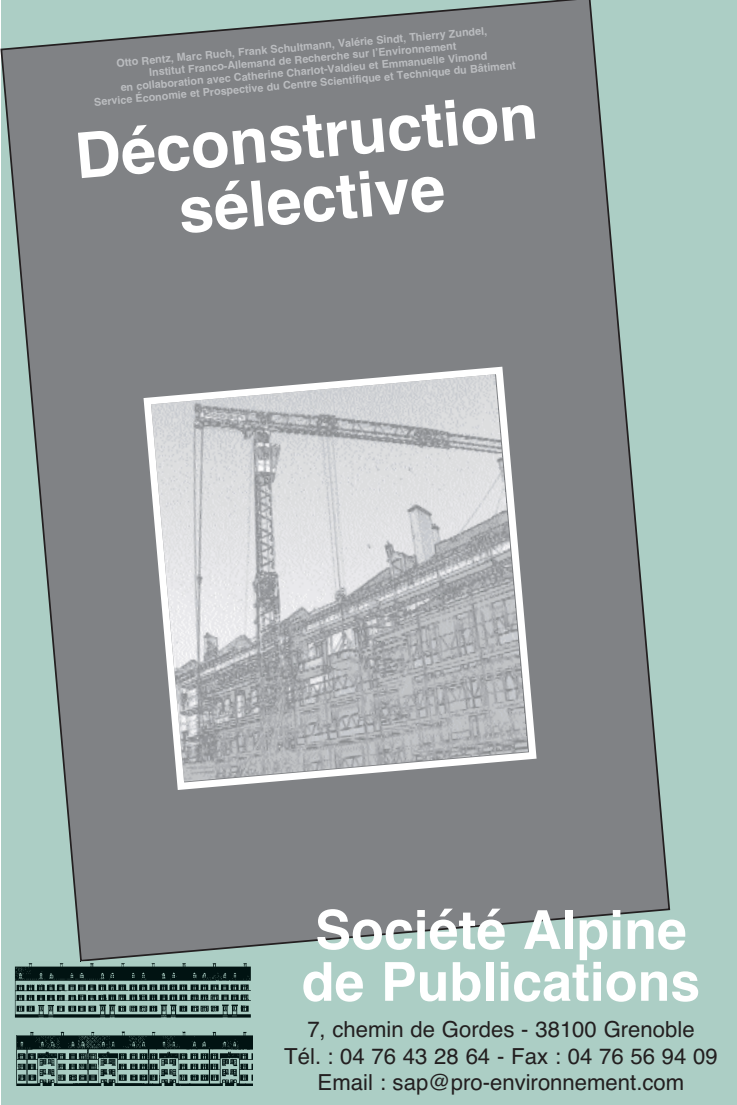

